\title{
Establishment of platform for screening insulin-like growth factor-1 receptor inhibitors and evaluation of novel inhibitors
}

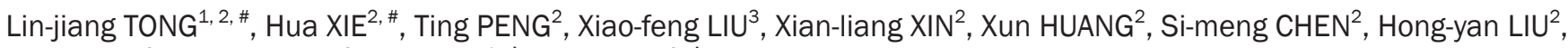 \\ Hong-lin $\mathrm{LI}^{3}$, Mei-yu GENG ${ }^{2}$, Ming YIN ${ }^{1, *}$, Jian $\mathrm{DING}^{2, *}$ \\ ${ }^{1}$ School of Pharmacy, Shanghai Jiao Tong University, Shanghai 200240, China; ${ }^{2}$ State Key Laboratory of Drug Research, Division of \\ Antitumor Pharmacology, Shanghai Institute of Materia Medica, Chinese Academy of Sciences, Shanghai 201203, China; ${ }^{3}$ Shanghai \\ Key Laboratory of Chemical Biology, School of Pharmacy, East China University, Shanghai 200237, China
}

Aim: The insulin-like growth factor-1 receptor (IGF1R) is over-expressed in a wide variety of tumors and contributes to tumor cell proliferation, metastasis and drug resistance. The aim of this study was to establish a sensitive screening platform to identify novel IGF1R inhibitors.

Methods: The catalytic domain of IGF1R was expressed using the Bac-to-Bac baculovirus expression system. The screening platform for IGF1R inhibitors was established based on ELISA. The binding profile of IGF1R with the inhibitors was predicted with molecular docking and then subjected to the surface plasmon resonance (SPR) approach. The growth inhibition of cancer cells by the inhibitors was assessed with MTT assay. Apoptosis was analyzed using flow cytometry and Western blotting.

Results: A naturally occurring small molecule compound hematoxylin was identified as the most potent inhibitor $\left(\mathrm{IC}_{50}\right.$ value $=1.8 \pm 0.1$ $\mu \mathrm{mol} / \mathrm{L}$ ) within a library of more than 200 compounds tested. Molecular simulation predicted the possible binding mode of hematoxylin with IGF1R. An SPR assay further confirmed that hematoxylin bound directly to IGF1R with high binding affinity $\left(\mathrm{Kd}=4.2 \times 10^{-6} \mathrm{~mol} / \mathrm{L}\right)$. In HL-60 cancer cells, hematoxylin inactivated the phosphorylation of IGF1R and downstream signaling and therefore suppressed cell proliferation. Mechanistic studies revealed that hematoxylin induced apoptosis in HL-60 cells via both extrinsic and intrinsic pathways. Conclusion: A simple, sensitive ELISA-based screening platform for identifying IGF1R inhibitors was established. Hematoxylin was identified as a promising IGF1R inhibitor with effective antitumor activity that deserves further investigation.

Keywords: insulin like growth factor 1 receptor (IGF1R); anticancer drug; enzyme-linked immunosorbent assay (ELISA); hematoxylin; cell proliferation; apoptosis; HL-60 cell line

Acta Pharmacologica Sinica (2011) 32: 930-938; doi: 10.1038/aps.2011.23; published online 6 Jun 2011

\section{Introduction}

Insulin-like growth factor 1 receptor (IGF1R) belongs to the insulin receptor (IR) family of receptor tyrosine kinase and is composed of two $\alpha$ and two $\beta$ subunits $^{[1,2]}$. The extracellular a subunit is responsible for ligand binding, whereas the $\beta$ subunit consists of a transmembrane domain and a cytoplasmic tyrosine kinase domain. The receptor is predominantly activated by IGF-I and IGF-II, but it can also be activated by insulin at a much lower affinity (500-1000 fold less). Ligand binding activates intrinsic tyrosine kinase activity, resulting in trans- $\beta$ subunit autophosphorylation and stimulation of

\footnotetext{
\# These authors contributed equally to this work.

* To whom correspondence should be addressed.

E-mail suozhang@mail.shcnc.ac.cn (Jian DING); myin@sjtu.edu.cn (Ming YIN)

Received 2010-11-24 Accepted 2011-03-07
}

signaling cascades, including phosphatidylinositol 3-kinase (PI3K)/ AKT and mitogen-activated protein kinase (MAPK) pathways. Activation of these pathways has been reported to result in cellular proliferation, survival, transformation, metastasis, and angiogenesis. Deregulation of the IGF1/IGF1R axis has been implicated in the development and progression of several cancers, including myeloma, breast, prostate, colon, ovarian, and lung cancer ${ }^{[3]}$. Increased expression of IGF1R and its ligands correlates with poor prognosis, reduced survival and drug resistance. Thus, the IGF1/IGF1R axis has become a promising target for anticancer drug development ${ }^{[4,5]}$.

Several approaches have been employed to target IGF1R, which include monoclonal antibodies, antisense oligonucleotides, IGF1R siRNA, and small molecule inhibitors. To date, more than 25 candidates have been identified at various stages of development; these candidates are predominantly small 
molecule inhibitors and monoclonal antibodies that target IGF1R $^{[6]}$. For example, CP-751871 ${ }^{[7]}$, a fully human monoclonal antibody specific for IGF1R, is in phase III clinical trial as a component of combination therapies for the treatment of nonsmall cell lung cancer (NSCLC). Nordihydroguaiaretic acid Insm-18/ $\mathrm{NGDA}^{[8]}$, a dual inhibitor of IGF1R and human epidermal growth factor receptor 2 (HER2), is in phase II clinical trial for the treatment of prostate cancer. In addition, several small molecule IGF1R inhibitors are currently in clinical or preclinical development, including picropodophyllin (PPP) ${ }^{[9]}$, NVP-AEW541 ${ }^{[10]}$, AG-1024 $4^{[11]}$, BMS-536942 $2^{[12]}$, BMS-554417 $7^{[13]}$, and NVP-ADW742 ${ }^{[14]}$. However, none of these inhibitors has been approved by the FDA for clinical uses. Meanwhile, with an increasing number of compounds available for screening, tremendous efforts have been made toward finding novel inhibitors as anti-tumor candidates. These efforts highlight the importance of establishing an efficient screening platform to identify and develop novel IGF1R inhibitors.

Here, we established a simple and effective screening model to identify potential IGF1R inhibitors. Several novel inhibitors were identified from the library, and hematoxylin stood out as a distinct compound that can suppress IGF1R activities both at an enzymatic level and in intact cells and inhibit the proliferation of HL-60 human cancer cells. Moreover, hematoxylin induces caspase-dependent apoptosis in cancer cells via both extrinsic and intrinsic pathways.

\section{Materials and methods Agents}

A compound library is maintained in our laboratory. I-OMeAG 538, a known IGF1R inhibitor, was purchased from Calbiochem (San Diego, CA, USA). Stock solutions of the compounds were prepared in DMSO at a concentration of 10 $\mathrm{mmol} / \mathrm{L}$, stored in aliquots at $-20^{\circ} \mathrm{C}$, and thawed immediately prior to use. Insulin receptor tyrosine kinase was purchased from Millipore.

\section{Cell culture}

The Spodoptera frugiperda (Sf9) and Trichoplusia ni (T ni) insect cell lines were kind gifts from Dr Jia LI (National Center for Drug Screening, Chinese Academy of Sciences, Shanghai, China) and Prof Xiang-fu WU (Institute of Biochemistry and Cell Biology, Chinese Academy of Sciences, Shanghai, China), respectively. Cells were cultured in modified TNM-FH medium (Sigma) supplemented with 10\% fetal bovine serum (Gibco-BRL) and $50 \mu \mathrm{g} / \mathrm{mL}$ gentamicin. Cultures were grown as monolayers in stationary flasks at $27^{\circ} \mathrm{C}$.

The HL-60 human leukemia cell line was obtained from the American Type Culture Collection (Rockville, MD, USA) and cultured in RPMI-1640 (Gibco-BRL) supplemented with 10\% fetal bovine serum, $4 \mathrm{mmol} / \mathrm{L}$ glutamine, 100 units/mL penicillin and $100 \mu \mathrm{g} / \mathrm{L}$ streptomycin in a humidified atmosphere of $95 \%$ air and $5 \% \mathrm{CO}_{2}$ at $37^{\circ} \mathrm{C}$.

\section{Cloning and expression of the IGF1R catalytic domain}

Full length human IGF1R was purchased from Addgene. The catalytic domain (amino acids 999-1274) of IGF1R (IGF1R-CD) was obtained by PCR. The primers used for amplification included sense (5'-CGCGGATCCGATCACCATGAGCCGGGAAC-3') and antisense (5'-CCGGAATTCAAGGAGACCTCCCGGAAGC-3') oligonucleotides containing BamH I and EcoR I sites, respectively. The PCR reaction was performed in a Peltier Thermal Cycler-200 with Pyrobest DNA Polymerase (TaKaRa Biotechnology Co, Ltd) using the following conditions: $94^{\circ} \mathrm{C}$ for $5 \mathrm{~min}$, then 30 cycles of $94{ }^{\circ} \mathrm{C}$ for $1 \mathrm{~min}, 55^{\circ} \mathrm{C}$ for $1 \mathrm{~min}, 72{ }^{\circ} \mathrm{C}$ for $3 \mathrm{~min}$, followed by a final elongation at $72{ }^{\circ} \mathrm{C}$ for $10 \mathrm{~min}$. Amplified cDNA was digested with $\mathrm{BamH}$ $\mathrm{I} /$ EcoR I and subcloned into the corresponding restriction sites of pAcGP67-A (Invitrogen). The correct cDNA was confirmed by DNA sequencing. Recombinant Bacmid DNA was prepared following the Bac-to-Bac baculovirus expression system manual (Invitrogen) and subsequently transfected into $\mathrm{T}$ ni cells using the CELLFECTIN reagent (Invitrogen). Transfection mixtures were removed after a $5 \mathrm{~h}$ incubation, and TNM-FH medium was added. Culture supernatants containing baculovirus were harvested at $72 \mathrm{~h}$ postinfection by centrifugation at $500 \times g$ for $5 \mathrm{~min}$ and transferred into a fresh tube. Next, T ni cells were infected with the baculovirus at $27^{\circ} \mathrm{C}$ for $72 \mathrm{~h}$, when expression levels of IGF1R were highest. T ni cells were harvested at $4^{\circ} \mathrm{C}$ at $500 \times g$ for 5 min and washed with ice-cold phosphate buffered saline (PBS). Finally, cells were re-spun as above and quickly frozen at $-80{ }^{\circ} \mathrm{C}$ after discarding the supernatant.

\section{Purification of IGF1R-CD from T ni cells}

The desired amount of Ni-NTA resin (QIAGEN) was packed into a disposable column (QIAGEN), which was equilibrated as recommended by the manufacturer. $\mathrm{T}$ ni cell pellets were resuspended and lysed in ice-cold lysis buffer $[50 \mathrm{mmol} / \mathrm{L}$ Tris- $\mathrm{HCl}$ (pH 8.5), $5 \mathrm{mmol} / \mathrm{L}$ 2-mercaptoethanol, $100 \mathrm{mmol} / \mathrm{L}$ $\mathrm{KCl}, 1 \mathrm{mmol} / \mathrm{L} \mathrm{PMSF}$, and 1\% Nonidet P-40]. Cells were sonicated (15 s pulses at $30 \mathrm{~s}$ intervals with an output power level of $10 \mathrm{~W}$ ), cell lysates were centrifuged at $10000 \times \mathrm{g}$ for $10 \mathrm{~min}$ at $4{ }^{\circ} \mathrm{C}$, and supernatants were loaded onto a preequilibrated column. The column was washed with ice-cold buffer A [20 $\mathrm{mmol} / \mathrm{L}$ Tris- $\mathrm{HCl}$ (pH 8.5), $500 \mathrm{mmol} / \mathrm{L} \mathrm{KCl}, 20 \mathrm{mmol} / \mathrm{L}$ imidazole, $5 \mathrm{mmol} / \mathrm{L} 2$-mercaptoethanol, and 10\% (v/v) glycerol] and buffer B [20 mmol/L Tris- $\mathrm{HCl}(\mathrm{pH} 8.5), 1 \mathrm{~mol} / \mathrm{L} \mathrm{KCl}, 5$ $\mathrm{mmol} / \mathrm{L} 2$-mercaptoethanol, and 10\% (v/v) glycerol] until the $O D_{280}$ returned to baseline. Proteins were eluted with ice-cold buffer C [20 mmol/L Tris-HCl (pH 8.5), 100 mmol/L KCl, 100 $\mathrm{mmol} / \mathrm{L}$ imidazole, $5 \mathrm{mmol} / \mathrm{L} 2$-mercaptoethanol, and $10 \%$ $(v / v)$ glycerol]. The active fraction containing the enzyme was divided into aliquots, rapidly frozen, and stored at $-80^{\circ} \mathrm{C}$.

\section{Protein determination and detection}

Protein concentrations were determined using the BCA Kit method. To identify proteins expressed in the T ni cells, cell extracts, flow-through samples and purified proteins were resolved on 10\% SDS-polyacrylamide gels and either stained with Coomassie blue dye or gently rocked with an anti-HisTag antibody overnight at $4{ }^{\circ} \mathrm{C}$. In the latter procedure, the 
antibody-protein complexes were captured on protein A agarose beads (Boehringer Mannheim) for $2 \mathrm{~h}$ at $4{ }^{\circ} \mathrm{C}$, and the proteins were then boiled off in the presence of $D L$-dithiothreitol (DTT). After the proteins were separated by electrophoresis and transferred to a nitrocellulose membrane (Amersham Life Sciences), the blot was probed with an anti-IGF1R antibody (Upstate Biotechnology) and visualized with an enhanced chemiluminescence detection reagent (Pierce).

\section{Protein kinase assays}

IGF1R-CD tyrosine kinase activity was determined by an enzyme-linked-immunosorbent assay (ELISA) in 96-well plates pre-coated with $2.5 \mu \mathrm{g} /$ well poly(Glu, Tyr $)_{4: 1}$ (Sigma) as a substrate. Fifty microliters of $10 \mu \mathrm{mol} / \mathrm{L}$ ATP solution diluted in reaction buffer [ $50 \mathrm{mmol} / \mathrm{L}$ HEPES, $\mathrm{pH}$ 7.4, 20 $\mathrm{mmol} / \mathrm{L} \mathrm{MgCl}_{2}, 0.1 \mathrm{mmol} / \mathrm{L} \mathrm{Na}_{3} \mathrm{VO}_{4}$, and $1 \mathrm{mmol} / \mathrm{L} \mathrm{DTT]}$ was added to each well, and the reaction was initiated by the addition of increasing concentrations of IGF1R tyrosine kinase. After incubation for $1 \mathrm{~h}$ at $37^{\circ} \mathrm{C}$, the plate was washed three times with phosphate buffered saline containing $0.1 \%$ Tween 20 (PBST). Next, $100 \mu \mathrm{L}$ of antiphosphotyrosine (PY99; 1:1000 dilution) antibody was added. After $0.5 \mathrm{~h}$ incubation at $37^{\circ} \mathrm{C}$, the plate was washed three times and goat anti-mouse IgG horseradish peroxidase $(100 \mu \mathrm{L}$ of a 1:2000 dilution) diluted in PBST containing $5 \mathrm{mg} / \mathrm{mL}$ BSA was added. The plate was reincubated at $37^{\circ} \mathrm{C}$ for $0.5 \mathrm{~h}$ and washed as before. Finally, $100 \mu \mathrm{L}$ of color development solution $\left(0.03 \% \mathrm{H}_{2} \mathrm{O}_{2}\right.$ and 2 $\mathrm{mg} / \mathrm{mL}$ o-phenylenediamine in $0.1 \mathrm{~mol} / \mathrm{L}$ citrate buffer, $\mathrm{pH}$ 5.4) was added and the plate was incubated at room temperature until color emerged. The reaction was terminated by the addition of $50 \mu \mathrm{L}$ of $2 \mathrm{~mol} / \mathrm{L} \mathrm{H}_{2} \mathrm{SO}_{4}$, and the $A_{492}$ was measured using a multiwell spectrophotometer (SpectraMax 190, Molecular Devices). Similar assays were performed with varying concentrations of IGF1R-CD tyrosine kinase, ATP, Poly (Glu, Tyr $)_{4: 1}, \mathrm{Mg}^{2+}$, and $\mathrm{Mn}^{2+}$. IR tyrosine kinase activity was detected by ELISA as described above.

\section{Screening of IGF1R inhibitors}

The IGF1R inhibitors were screened in 96-well plates precoated with $2.5 \mu \mathrm{g} /$ well poly(Glu, Tyr $)_{4: 1}$. Each well was treated with $50 \mu \mathrm{L}$ of $10 \mu \mathrm{mol} / \mathrm{L}$ ATP solution and $1 \mu \mathrm{L}$ of compound at varying concentrations. The known IGF1R inhibitor, I-OMe-AG 538, was used as a positive control, and $0.1 \%(v / v)$ DMSO was utilized as the negative control. Experiments at each concentration were performed in duplicate. The reaction was initiated by adding $49 \mu \mathrm{L}$ of IGF1R-CD diluted in reaction buffer, and the $A_{492}$ was measured as described above. The inhibition rate (\%) was calculated using the equation [1$\left(A_{492 \text { treated }} / A_{492 \text { control })}\right) \times 100 \%$. The $\mathrm{IC}_{50}$ values were determined from the results of at least three independent tests.

\section{Surface plasmon resonance (SPR) analysis}

The kinetics and specificity of the binding reactions between hematoxylin and IGF1R were performed with the ProteOn XPR36 (Bio-Rad). Briefly, IGF1R was immobilized on GLM sensor chips by amine coupling according to the protocol described in the application handbook. To assess real-time binding capacity, $100 \mu \mathrm{L}$ of soluble hematoxylin was injected over the sensor chip surface with the immobilized IGF1R and then washed with HBS-EP buffer for 5 min. The sensor chip surface was then regenerated using $60 \mu \mathrm{L} \mathrm{NaCl}(2 \mathrm{~mol} / \mathrm{L})$. All binding experiments were performed at $25^{\circ} \mathrm{C}$ with a constant flow rate of $50 \mu \mathrm{L} / \mathrm{min}$ PBST. For binding affinity assessment, the association phase was allowed to proceed to equilibrium. To correct for nonspecific binding and bulk refractive index change, a blank channel (FC2) without IGF1R was employed as a control for each experiment. Sensorgrams for all binding interactions were recorded in real time and analyzed after subtracting the values from the blank channel. Changes in mass due to the binding response were recorded as resonance units (RU). Binding kinetics and affinities were determined with ProteOn software 2.1.1.

\section{Molecular docking}

The 3D conformation of hematoxylin was generated based on the 2D structures from PubChem (http:// pubchem.ncbi.nlm. nih.gov/, accessed on 2009.12.11) (id 10603) using LigPrep (version 2.0, Schrödinger, LLC, NY, USA, 2005), and all of the stereo configurations were manually checked. The crystal structure of IGF1R (PDB code 1IR3) was prepared with the Protein Preparation Wizard workflow of Schrodinger (version 1.0, Schrödinger, LLC, NY, USA, 2005) with all crystal water molecules and binding ATP removed and all hydrogen atoms added. Molecular docking was performed using Glide (version 4.0, Schrödinger, LLC, NY, USA, 2005). The grid-enclosing box was centered on the centroid of co-complexed ligand (ANP) to enclose residues located within $20.0 \AA$ around the ATP binding site, and a scaling factor of 1.0 was set to van der Waals (VDW) radii of those receptor atoms with the partial atomic charge less than 0.25 . The extra-precision (XP) mode of Glide was used, and the top ranked poses of hematoxylin (ranked by lowest Glide Score) were retained after visual analysis.

\section{In vitro antiproliferation assay}

Cell proliferation was evaluated using the MTT assay as previously described ${ }^{[15]}$. Briefly, cells were seeded into 96-well plates and grown for $24 \mathrm{~h}$. The cells were then treated with or without different concentrations of hematoxylin and grown for a further $72 \mathrm{~h}$. At the end of exposure, $20 \mu \mathrm{L}$ of MTT $(5 \mathrm{~g} / \mathrm{L})$ was added to each well and the plates were incubated at $37^{\circ} \mathrm{C}$ for $4 \mathrm{~h}$. Then a "triplex solution" (10\% SDS-5\% isobutanol-12 $\mathrm{mmol} / \mathrm{L} \mathrm{HCl}$ ) was added and the plates were incubated at $37^{\circ} \mathrm{C}$ for $12 \mathrm{~h}$. The plates were then measured at $570 \mathrm{~nm}$ using a multiwell spectrophotometer (SpectraMax 190, Molecular Devices). The cell growth inhibition rate was calculated by the equation $\left[1-\left(A_{570}\right.\right.$ treated $/ A_{570}$ control $\left.)\right] \times 100 \%$. The $\mathrm{IC}_{50}$ value was obtained by the Logit method and was determined from the results of at least 3 independent tests.

DAPI staining was performed as described previously ${ }^{[16]}$. 
Briefly, HL-60 cells $\left(3 \times 10^{5}\right.$ cells $\left./ \mathrm{mL}\right)$ were seeded into six-well plates and treated with different concentrations of hematoxylin for the indicated times. The cells were harvested and fixed with $4 \%$ paraformaldehyde for $30 \mathrm{~min}$ at room temperature. After cells were washed with PBS by centrifugation at $1000 \times g$ for $5 \mathrm{~min}, 2 \mu \mathrm{L}$ DAPI $(5 \mu \mathrm{g} / \mathrm{mL})$ was added to the fixed cells for $5 \mathrm{~min}$, after which they were examined by fluorescence microscopy. Apoptotic cells were identified by condensation and fragmentation of chromatin.

\section{Flow cytometry assay}

To evaluate the apoptosis-inducing activity of hematoxylin, HL-60 cells were plated at a density of $3 \times 10^{5}$ cells $/ \mathrm{mL}$ into sixwell plates and incubated with or without the indicated concentrations of hematoxylin for $24 \mathrm{~h}$. The cells were then harvested and fixed in $70 \%$ ethanol and stored at $4{ }^{\circ} \mathrm{C}$ overnight. Afterward, the cells were stained in PBS containing $40 \mu \mathrm{g} / \mathrm{mL}$ RNase and $10 \mu \mathrm{g} / \mathrm{mL}$ propidium iodide (PI) at room temperature in the dark for $30 \mathrm{~min}$. Subsequently, the cells were analyzed using a FACS-Calibur cytometer (Becton Dickinson, San Jose, CA, USA). The cells undergoing apoptosis were obtained from the distinct sub- $\mathrm{G}_{1}$ region of the DNA distribution histograms. At least 10000 events were counted for each sample.

\section{Western blot analysis}

HL-60 cells $\left(3 \times 10^{5}\right.$ cells $\left./ \mathrm{mL}\right)$ were seeded into six-well plates and exposed to hematoxylin at various concentrations for $24 \mathrm{~h}$. After treatment, the cells were collected and suspended in lysis buffer (100 mmol/L Tris-HCl, pH 6.8, $200 \mathrm{mmol} / \mathrm{L}$ DTT, $4 \%$ SDS, $0.2 \%$ bromophenol blue, and $20 \%$ glycerol). The cell lysate was cleared by centrifugation at 14000 revolutions per minute for $15 \mathrm{~min}$. Lysate proteins were resolved by $7.5 \%$ sodium dodecyl sulfate polyacrylamide gel electrophoresis (SDS-PAGE) and transferred to nitrocellulose membranes. The membranes were incubated for $1 \mathrm{~h}$ in $5 \%$ milk, followed by $2 \mathrm{~h}$ of incubation with primary antibodies. The membranes were washed three times with PBS (with 0.1\% Tween-20) and then incubated with the respective peroxidase-conjugated secondary antibody for $1 \mathrm{~h}$. The membranes were washed again, developed using enhanced chemiluminescence (ECL, Amersham Biosciences), and exposed to Kodak X-Omat BT film.

\section{Statistical analysis}

Data shown were mean $\pm S D$ from three independent experiments.

\section{Results}

\section{Expression and purification of human IGF1R-CD}

The catalytic domain of the human IGF1R cDNA was subcloned into the pAcGP67-A vector for generation of a histidine (His) $)_{6}$-tagged fusion protein. The proteins were purified and detected as described in Materials and methods. The timecourse analysis of protein expression revealed maximal levels at $3 \mathrm{~d}$ post-infection (data not shown), and the protein concentration was approximately $1.5 \mathrm{~g} / \mathrm{L}$. The (His) ${ }_{6}$-IGF1R-CD fusion protein was observed as a major band migrating with an apparent molecular weight of $31 \mathrm{kDa}$ (Figure 1A), which is consistent with the expected size of the protein. When the elution fraction was probed by an antibody specifically raised against IGF1R, the corresponding immunoreactive band was detected with the same molecular weight (Figure 1B).

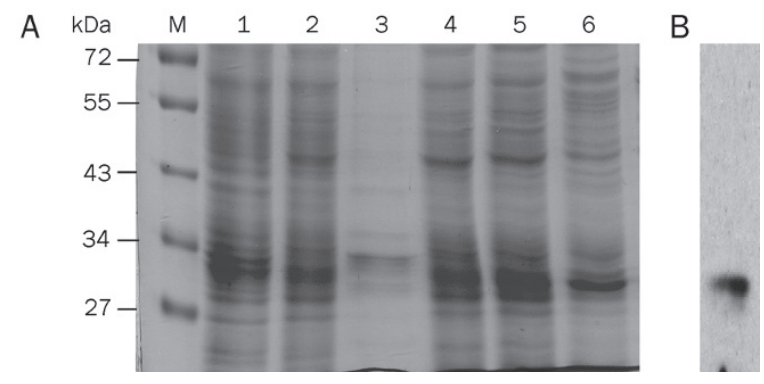

Figure 1. SDS-polyacrylamide gel analysis of purified IGF1R-CD. (A) IGF1R-CD was Ni-NTA column purified from $T$ ni insect cells, and the purity of the fusion protein was examined in aliquots from different steps of the purification. Lane 1, whole infected cell lysate; Lane 2, supernatant sample; Lane 3, cell debris after lysis; Lane 4, wash step fraction; Lane 5, elution step fraction 1; Lane 6, elution step fraction 2; M, molecular weight marker. Proteins were separated by $15 \%$ SDS-PAGE, and stained with Coomassie blue dye. (B) Parallel samples were immunoprecipitated using a polyclonal antibody against His-Tag and then Western blotted with an anti-IGF1R antibody.

\section{Kinase activity of IGF1R-CD}

To determine the kinase activity of IGF1R-CD and the kinase concentration required for the phosphorylation of substrate, we used an ELISA assay. The peptide substrate employed in this test is a random copolymer of glutamic acid and tyrosine [poly(Glu, Tyr $\left.)_{4: 1}\right]^{[17]}$. Varying quantities of IGF1R-CD were mixed with a fixed amount of ATP $(5 \mu \mathrm{mol} / \mathrm{L})$ and then added to 96 -well plates pre-coated with $20 \mu \mathrm{g} / \mathrm{mL}$ of poly(Glu, Tyr $)_{4: 1}$. As illustrated in Figure 2A, the phosphorylation state of the substrates increased with rising concentrations of IGF1R-CD protein kinase, reaching a high level $\left(A_{490}=1.0\right)$ upon exposure to $0.75 \mu \mathrm{g}$ of IGF1R-CD protein kinase. Consequently, $0.75 \mu \mathrm{g}$ of IGF1R-CD protein kinase per well was used as a defined concentration in the subsequent screening. Next, we investigated the effects of the concentrations of both ATP and the peptide substrate on the phosphorylation reaction. The results in Figure $2 \mathrm{~B}$ and $2 \mathrm{C}$ clearly demonstrated a positive correlation between kinase activity and the substrate concentrations, releasing the optimal concentrations of ATP and poly(Glu, Tyr $)_{4: 1}$ in the kinase assay at $5 \mu \mathrm{mol} / \mathrm{L}$ and 20 $\mu \mathrm{g} / \mathrm{mL}$, respectively.

\section{Divalent cation is required for substrate phosphorylation}

Substrate phosphorylation by a kinase requires the presence of divalent cations. The interactions among these factors are complex. The kinases are either activated or inactivated upon binding to such divalent cations as $\mathrm{Mg}^{2+}$ or $\mathrm{Mn}^{2+}$. ATP usually coordinates with $\mathrm{Mg}^{2+}$ and $\mathrm{Mn}^{2+}$ to form ATP-Mg and ATP- 

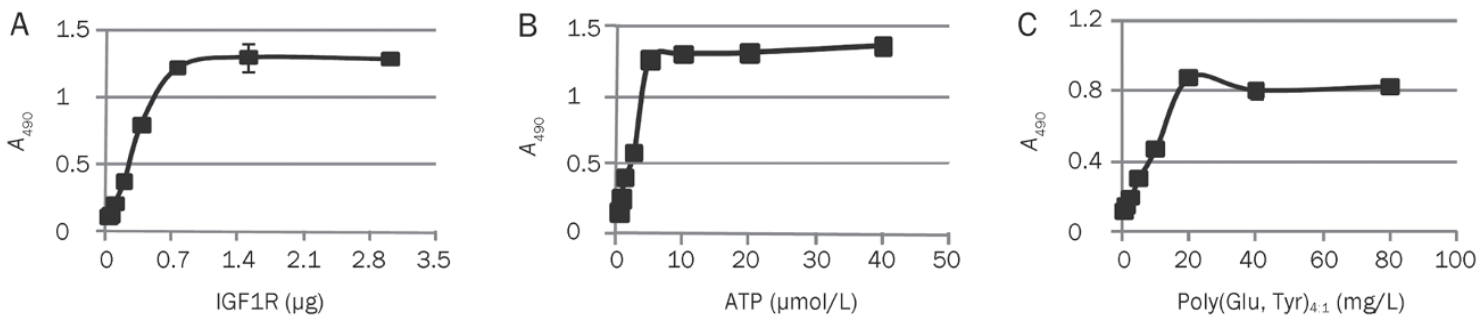

Figure 2. ELISA assays determining the phosphorylation of a synthetic peptide. (A) Plot of $A_{490}$ vs IGF1R quantity illustrating the relationship between substrate phosphorylation status and IGF1R level. (B) Plot of $A_{490}$ vs ATP concentration, and (C) Plot of $A_{490}$ vs concentration of synthetic substrate poly(Glu,Tyr $)_{4: 1}$, used to determine the optimal concentration of substrate in the screening model. Data shown were mean \pm SD from three independent experiments.

Mn, which serve as the actual substrates in phosphorylation. Accordingly, we examined the effects of divalent cation concentrations on the rate of substrate phosphorylation catalysis by IGF1R-CD. As shown in Figure 3A and 3B, with increases in their concentrations, the kinase activities were increased accordingly within a certain range of $\mathrm{Mg}^{2+}$ or $\mathrm{Mn}^{2+}$ concentration. Optimal kinase activity was observed at $10 \mathrm{mmol} / \mathrm{L}$ and

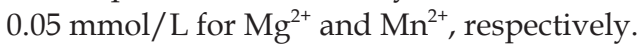

\section{Screening of IGF1R kinase inhibitors}

We have identified the optimal concentration of key factors in our screening model. We then used the compound I-OMe-AG 538, a well known IGF1R inhibitor, as the positive control to determine the efficiency of the model. As illustrated in Figure 3C, I-OMe-AG 538 inhibited the IGF1R-CD kinase activity in a concentration-dependent manner, with an $\mathrm{IC}_{50}$ value of 0.175 $\mu \mathrm{mol} / \mathrm{L}$. These data are consistent with a previous report ${ }^{[18]}$, illustrating that the model established in the present study can be used for the identification of IGF1R inhibitors.

The optimized IGF1R-based ELISA assay was then used to screen more than 200 compounds featuring a wide variety of chemical structures. The primary screening was performed at a single concentration of $10 \mu \mathrm{mol} / \mathrm{L}$ of each compound. Subsequently, all primary hits demonstrating at least 30\% inhibition relative to control were retested, and the $\mathrm{IC}_{50}$ values were calculated. Eventually, eight potential inhibitors were characterized (data not shown). Notably, hematoxylin, a widely used nuclear dye, was revealed to be one of the most potent inhibitors of IGF1R. This compound effectively inhibited the
IGF1R activity in a dose-dependent manner, with an $\mathrm{IC}_{50}$ value of $1.8 \pm 0.1 \mu \mathrm{mol} / \mathrm{L}$ (Figure $4 \mathrm{~A}$ ).

\section{Hematoxylin binds to the kinase domain of IGF1R}

Because hematoxylin was identified as a novel IGF1R inhibitor, we then wanted to know whether its inhibitory activity on IGF1R was associated with direct engagement with tyrosine kinases. An SPR assay was employed to assess the interaction between hematoxylin and IGF1R. For this assay, hematoxylin was immobilized on CM-5 sensor chips. The response of binding units of hematoxylin to IGF1R was recorded as described in Materials and methods. As shown in Figure 4B, hematoxylin was noted to bind to IGF1R in a concentrationdependent manner with high affinity, yielding the equilibrium dissociation constant $(\mathrm{Kd})$ of $4.2 \times 10^{-6} \mathrm{~mol} / \mathrm{L}$. The results demonstrated that hematoxylin could bind directly to IGF1R-CD.

\section{Docking analysis of hematoxylin with IGF1R tyrosine kinase domain}

To further explore the binding profiles of the compound, a binding model of hematoxylin with the IGF1R tyrosine kinase domain was simulated by molecular docking. The results in Figure $4 \mathrm{C}$ indicated that hematoxylin occupies the adenine pocket of the ATP binding site of IGF1R. The binding orientation of one catechol moiety is located at the hinge region through hydrogen bonds with the backbone of the conserved residues among the kinase family (Glu 1077 and Met 1079). Two hydrogen bonds exist between the other catechol moiety and carboxylates of Asp 1150 of the Asp-Phe-Gly (DFG) motif
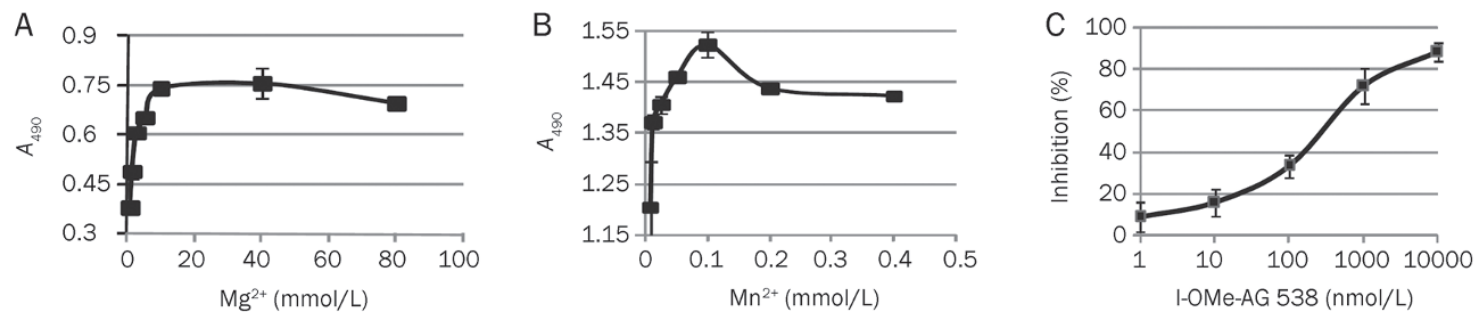

Figure 3. Titration of the divalent cations and determination of IGF1R inhibitory activity of I-OMe-AG 538 in the established screening model. (A-B) Plot of $A_{490}$ vs concentrations of divalent cations, used to determine the metal requirement for catalysis of substrate phosphorylation. (C) Effect of positive IGF1R inhibitor, I-OMe-AG 538, on IGF1R tyrosine kinase activity. Data shown were mean \pm SD from three independent experiments. 
A

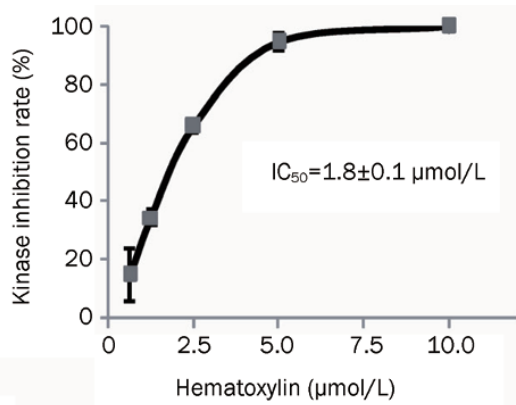

B

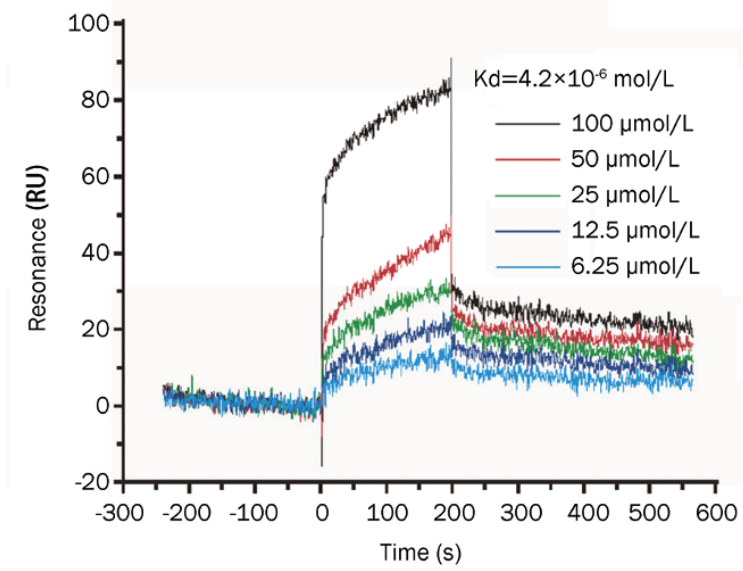

C

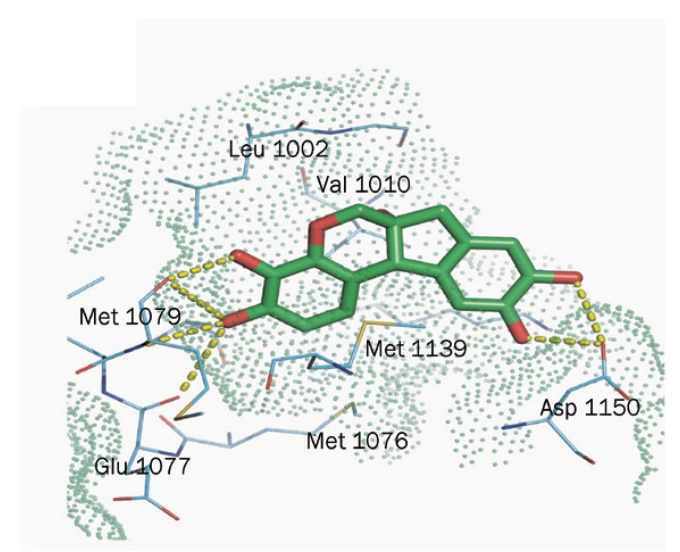

Figure 4. Hematoxylin inhibits IGF1R activity in vitro and binds directly to IGF1R-CD. (A) Inhibitory activity of hematoxylin on IGF1R detected by ELISA assay. Tests were performed three times independently. (B) Hematoxylin binds to IGF1R-CD. SPR assay was performed as described in Materials and methods. (C) The binding mode of hematoxylin to the kinase domain of IGF1R predicted by molecular docking. The ligand (green carbon) and residues (cyan carbon) are represented as sticks and lines, respectively. The yellow dashed lines denote the hydrogen bonds and the oxygen atoms are colored in red. The molecular surface of the binding site was shown as green dots. The structure figure was prepared using PyMol (www.pymol.org). Data shown were mean \pm SD from three independent experiments.

of the activation loop. Multiple hydrophobic interactions and van der Waals (VDW) contacts to the ATP binding site were also observed, including Leu 1002, Val 1010, Met 1139, and
Met 1076. The docking results indicated that the hematoxylin formed a good interaction with the IGF1R active site, which may underlie the direct binding of the compound to IGF1R.

Hematoxylin potently inhibits the kinase activity of the insulin receptor (IR) tyrosine kinase

Because the insulin receptor is highly homologous to IGF1R, we further determined the effect of hematoxylin on the kinase activity of IR using an ELISA assay. As shown in Table 1, hematoxylin potently inhibited the kinase activity of IR in vitro with an $\mathrm{IC}_{50}$ value of $0.67 \pm 0.10 \mu \mathrm{mol} / \mathrm{L}$, which is lower than that of hematoxylin on IGF1R. I-OMe-AG 538 was used as a positive control.

Table 1. Inhibitory effect of hematoxylin on the kinase activity of IR. Data shown were mean $\pm S D$ from three independent experiments.

\begin{tabular}{ll}
\hline Compound & $\mathrm{IC}_{50}(\mu \mathrm{mol} / \mathrm{L})$ \\
\hline Hematoxylin & $0.67 \pm 0.10$ \\
I-OMe-AG 538 & $0.21 \pm 0.12$ \\
\hline
\end{tabular}

Hematoxylin inhibits proliferation and IGF1R phosphorylation in HL-60 human leukemia cells

Next, we selected HL-60 human leukemia cells, which naturally express high levels of IGF1R, to test the inhibitory effects of hematoxylin on cell proliferation. As illustrated in Figure $5 \mathrm{~A}$, hematoxylin inhibited the proliferation of HL-60 cells in a concentration-dependent manner. Moreover, this compound inhibited IGF-induced IGF1R phosphorylation in a dose-dependent manner (Figure $5 \mathrm{~B}$ ), with the maximal effects obtained at $10 \mu \mathrm{mol} / \mathrm{L}$ of hematoxylin. Hematoxylin also decreased the phosphorylation of AKT and Erk1/2, the downstream signaling pathways of IGF1R. Together with the data described above, we conclude that hematoxylin at least partially inhibited cell proliferation via blocking the activation of IGF1R and subsequent downstream signaling.

\section{Hematoxylin induces apoptosis in HL-60 cells}

Because IGF1R signaling prevents apoptosis through the PI3KAKT pathway ${ }^{[19]}$, we next wanted to address whether hematoxylin induces apoptosis in HL-60 cells.

We first observed morphological changes in apoptosis induced by hematoxylin using DAPI staining. As illustrated in Figure 6A, treatment with hematoxylin at $20 \mu \mathrm{mol} / \mathrm{L}$ for $24 \mathrm{~h}$ induced marked apoptotic changes in HL-60 cells compared to the control cells, as evidenced by the appearance of fragmented chromatin and apoptotic bodies.

For further confirmation, we quantified apoptotic cells using flow cytometry analysis. As shown in Figure 6B, the fraction of cells in sub $G_{1} / G_{0}$ increased in a dose-dependent manner after hematoxylin treatment for $24 \mathrm{~h}$. Approximately $10 \%$ of the HL-60 cells underwent apoptosis upon exposure to hema- 

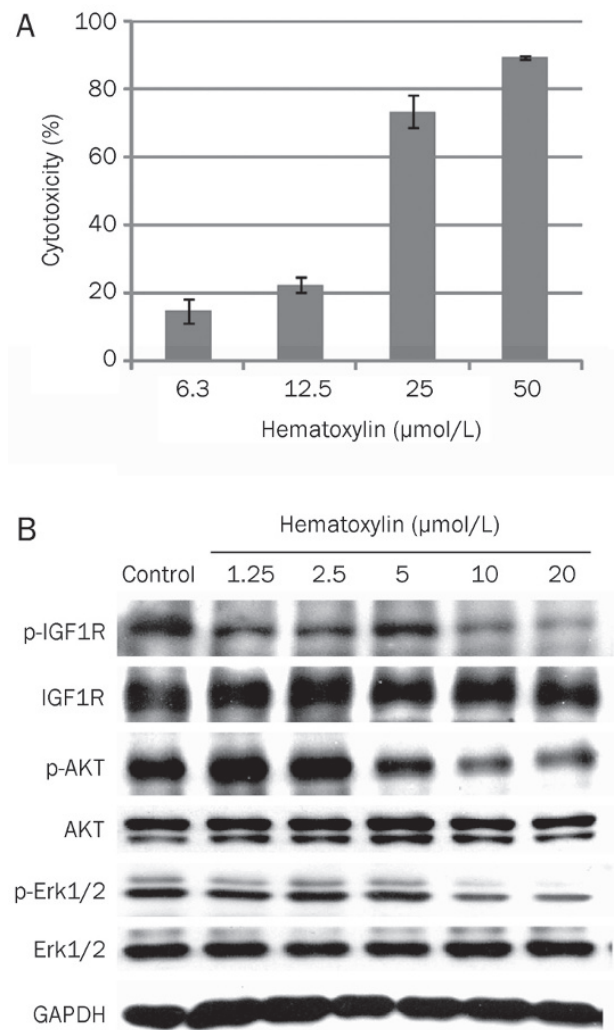

Figure 5. Effect of hematoxylin on the proliferation and the phosphorylation of IGF1R and downstream signaling pathways in HL-60 cells. (A) Growth inhibition of HL-60 cells by hematoxylin. Cells were treated with the indicated concentrations of hematoxylin for $72 \mathrm{~h}$. Cell viability was determined by sulforhodamine $B$ assay. The tests were repeated three times independently. (B) Hematoxlyin inhibits the phosphorylation of IGF1R and the activation of downstream signaling pathways in $\mathrm{HL}-60$ cells. Cells were cultured in the presence of different doses of hematoxylin for $24 \mathrm{~h}$ and harvested. Whole cell lysates were assayed for different proteins by immunoblotting. A representative anti-GAPDH immunoblot is shown as a loading control. Data shown were mean $\pm S D$ from three independent experiments.

toxylin $(1.25 \mu \mathrm{mol} / \mathrm{L})$, which increased to more than $23 \%$ with hematoxylin concentrations up to $20 \mu \mathrm{mol} / \mathrm{L}$.

Hematoxylin activates caspase $-3,-8$, and -9 and induces PARP cleavage

We further evaluated whether hematoxylin induced apoptosis in a caspase-dependent manner. The results clearly showed that caspase- 3 was activated after exposure to hematoxylin, as shown by a decrease in the procaspase- 3 band and an increase in the cleaved caspase-3 band (Figure 6C). In addition, hematoxylin exposure resulted in PARP cleavage, a downstream target of caspase, in a dose-dependent fashion (Figure 6C).

To gain further insights into the mechanisms involved in the activation of caspase- 3 , the effects of hematoxylin on caspase- 8 and caspase- 9 were preferentially determined. As shown in Figure 6C, we found that both caspase- 8 and caspase- 9 were obviously cleaved after hematoxylin treatment, suggesting the
A

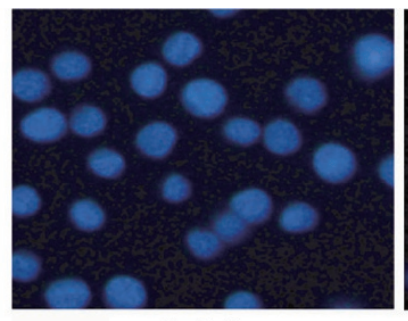

Control

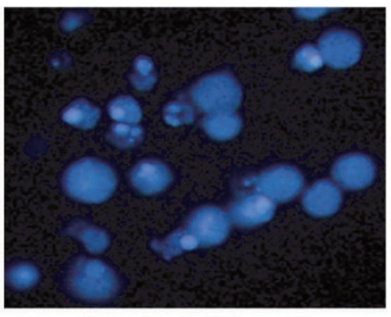

Hematoxylin $20 \mu \mathrm{mol} / \mathrm{L}$
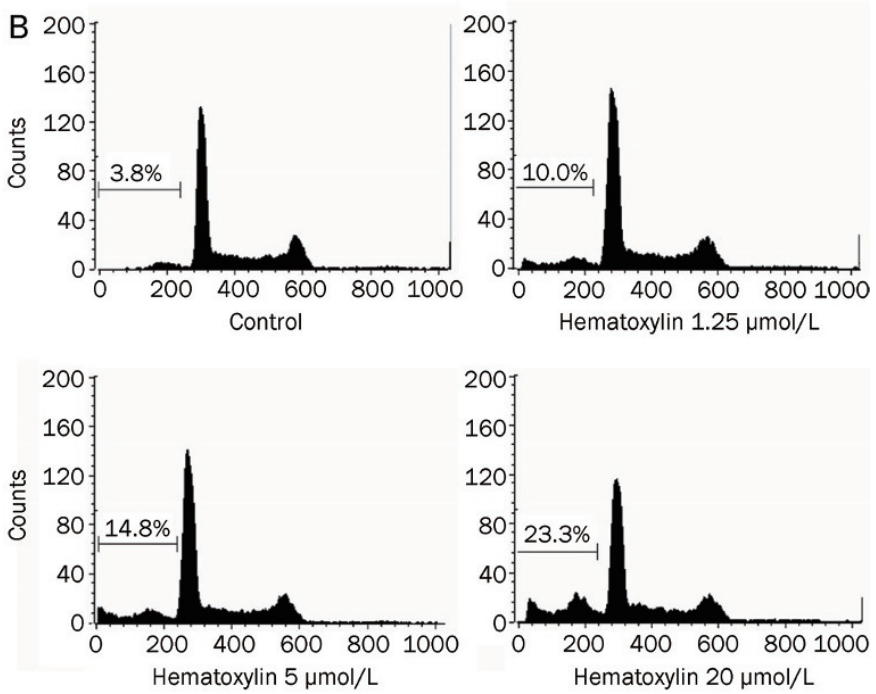

C

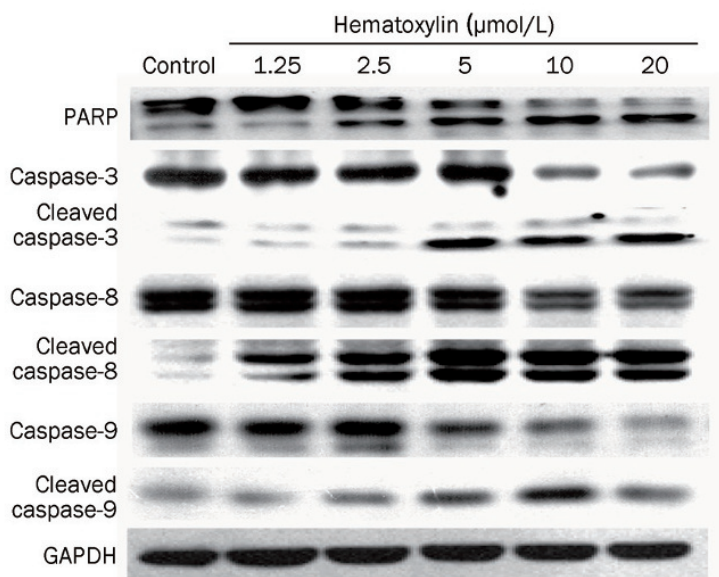

Figure 6. Hematoxylin induces apoptosis in HL-60 cells via both intrinsic and extrinsic apoptosis pathways. (A) DAPI staining assay. DAPI-stained nuclei of cells untreated or treated with $20 \mu \mathrm{mol} / \mathrm{L}$ hematoxylin were observed using a microcopy $(\times 200)$. (B) PI staining for flow cytometry. Cells were treated with hematoxylin for $24 \mathrm{~h}$ at indicated concentrations and then analyzed using FACS after they were fixed by $70 \%$ ethanol and stained with PI. Sub $G_{0} / G_{1}$ DNA content of cells were indicated. (C) Hematoxylin modulates the expression of apoptosis-related proteins. After treatment with or without desired concentration of hematoxylin for $24 \mathrm{~h}$, cells were collected and assayed by Western blotting analysis.

involvement of both the extrinsic and the intrinsic apoptosis pathways. 


\section{Discussion}

IGF1R signaling pathway is activated in many types of cancers due to aberrant expression of IGF1R or its ligands (such as IGF-I and IGF-II) and/or by decreased levels of the binding proteins. Disruption of IGF1R signaling is an attractive approach for blocking tumor growth and metastasis ${ }^{[1,3]}$. However, the efficient identification of novel IGF1R small molecule inhibitors primarily hinges on the availability of effective and feasible screening methods. Accumulating evidence has supported the notion that the catalytic domain accurately reflects the nature of the intact receptor. In the present study, the catalytic domain of IGF1R (IGF1R-CD) was successfully cloned and expressed using the Bac-to-Bac expression system. Using this active fragment, we developed a successful model for screening IGF1R inhibitors based on the phosphorylation of the IGF1R-CD substrate. ELISA, a widely used specific assay for the detection and quantification of antigens or antibodies without radioactive pollution, was employed in this assay. The reliability and feasibility of this method were validated by reference IGF1R inhibitors, followed by screening of 200 compounds for IGF1R inhibition activity. Of these compounds, eight compounds were identified as exhibiting inhibitory effects on IGF1R in the low micromolar to micromolar range. Hematoxylin, a well-known dye, stood out as a potent inhibitor of IGF1R at both the enzymatic and the cellular level. Molecular docking combined with SPR analysis revealed that hematoxylin binds to IGF1R with high affinity through hydrogen bonding to the hinge region of the ATP binding site and hydroxylation to the catalytic site.

Moreover, we found that hematoxylin exhibits potent inhibition of the IR kinase activity. IR is a receptor tyrosine kinase that is $84 \%$ homologous to the IGF1R kinase domain and $100 \%$ homologous in the ATP binding cleft. IR is primarily involved in the response to insulin and uptake of glucose into cells. Due to the high homology between the two receptors, inhibitors and antibodies directed at IGF1R often also affect IR signaling, resulting in potential side effects, such as diabetes and cardiotoxicity. However, insulin, acting via IR, has been recognized as able to stimulate proliferation of breast cancer cells, and increased levels of insulin have been related to an increased risk of different types of human cancer ${ }^{[20-22]}$. Moreover, IGF-II, the ligand of IGF1R, also binds with high affinity to IR and IGF1R/IR hybrid receptors. Thus, targeting IGF1R itself, but not IR, may not be adequate to inhibit IGF action. An ideal anti-IGF strategy would target both IGF1R and IR only in tumor cells and leave host IR in insulin target tissues (liver, muscle, and fat) unaffected ${ }^{[5,23]}$. Because our results demonstrate that hematoxylin can inhibit the kinase activities of both of IGF1R and IR, this compound deserves further investigation in vivo to evaluate target inhibition.

Activation of the IGF1R signaling pathway is involved in the growth, invasion and metastasis of cancer cells ${ }^{[5]}$. IGF1R has several survival signals, including PI3K/AKT/mTOR and Ras/MEK/MAPK pathways, which are able to protect cancer cells from apoptosis. Simultaneous inactivation of two of these pathways is required to inhibit the capacity of IGF1R to protect cancer cells from apoptotic injuries. In HL-60 human leukemia cancer cells, we found that hematoxylin inhibits the activation of IGF1R and downstream signaling. Importantly, such blockage of these signaling pathways eventually contributed to the subsequent induction of apoptosis and inhibition of cell proliferation. In addition, because our studies have proven that hematoxylin can inhibit multiple targets (including EGFR, FGFR, c-Met, and so on), inhibition of the downstream signaling pathways in HL-60 cells may result not only from the IGF1R inhibition but also from the inhibition of multiple kinases.

Detailed mechanistic studies indicated that caspases were involved in hematoxylin-induced apoptosis, as evidenced by the fact that hematoxylin is capable of activating caspase- 3 and the downstream target, PARP. Caspase-driven cascades are activated through the membrane death-receptor pathway utilizing caspase-8 or through the mitochondrial pathway relying on the activation of caspase- $9^{\left.9^{[24}, 25\right]}$. In congruence with this notion, we found that that both caspase- 8 and caspase- 9 were activated after hematoxylin treatment, suggesting that both the extrinsic and the intrinsic apoptosis pathways are involved in hematoxylin-induced apoptosis.

Several small molecular tyrosine kinase inhibitors (TKIs) have been launched into marketing and numerous candidates are under clinical or preclinical evaluation. However, several candidates have failed in clinical trials due to the complex nature of the crosstalk and signaling bypasses downstream of the targeted protein tyrosine kinase (PTK). PTK inhibitors with broad-spectrum specificity are increasingly expected to demonstrate a better clinical benefit than selective agents ${ }^{[26]}$. Some multi-targeted PTK inhibitors approved by the FDA/ EMEA, such as sorafenib ${ }^{[27]}$ and sunitinib ${ }^{[28]}$, are marked as a new generation of PTK inhibitor drugs. We have previously reported that hematoxylin is a multi-targeted inhibitor of several tyrosine kinases, including c-Src, c-Met, FGFR1, EGFR, VEGFR, PDGFR, and c-Kit ${ }^{[29]}$. These data, together with the newly recognized function of hematoxylin as a potent inhibitor of IGF1R, suggest that hematoxylin may be a promising therapeutic agent for the treatment of cancer.

In summary, we established an ELISA-based IGF1R inhibitor screening platform. Our new method will simplify the future identification of other IGF1R inhibitors, and using this approach, hematoxylin has emerged as a promising candidate for further investigation.

\section{Acknowledgements}

This work was financially supported by the Key New Drug Creation and Manufacturing Program (2009ZX09103-001 and 2009ZX09103-102), a grant from the Shanghai Science and Technology Committee (10431902600), and a grant from the Shanghai Key Lab of Chemical Biology (SKLCB-2008-03).

\section{Author Contribution}

Hua XIE, Ming YIN, and Jian DING designed research; Linjiang TONG, Hua XIE, and Ting PENG performed research; Xun HUANG, Si-meng CHEN, and Hong-yan LIU contributed 
the protein expression; Xian-liang XIN performed the SPR experiment; Xiao-feng LIU and Hong-lin LI performed the molecular docking; Hua XIE, Lin-jiang TONG, Mei-yu GENG, and Jian DING wrote the paper.

\section{References}

1 Laviola L, Natalicchio A, Giorgino F. The IGF-I signaling pathway. Curr Pharm Des 2007; 13: 663-9.

2 Adams TE, Epa VC, Garrett TP, Ward CW. Structure and function of the type 1 insulin-like growth factor receptor. Cell Mol Life Sci 2000; 57: 1050-93.

3 Pollak MN, Schernhammer ES, Hankinson SE. Insulin-like growth factors and neoplasia. Nat Rev Cancer 2004; 4: 505-18.

4 Samani AA, Yakar S, LeRoith D, Brodt P. The role of the IGF system in cancer growth and metastasis: overview and recent insights. Endocr Rev 2007; 28: 20-47.

5 Sachdev D, Yee D. Disrupting insulin-like growth factor signaling as a potential cancer therapy. Mol Cancer Ther 2007; 6: 1-12.

6 Dransfield DT, Cohen EH, Chang Q, Sparrow LG, Bentley JD, Dolezal O, et al. A human monoclonal antibody against insulin-like growth factorII blocks the growth of human hepatocellular carcinoma cell lines in vitro and in vivo. Mol Cancer Ther 2010; 9: 1809-19.

7 Iwasa T, Okamoto I, Suzuki M, Hatashita E, Yamada Y, Fukuoka M, et al. Inhibition of insulin-like growth factor 1 receptor by CP-751871 radiosensitizes non-small cell lung cancer cells. Clin Cancer Res 2009; 15: 5117-25.

8 Blecha JE, Anderson MO, Chow JM, Guevarra CC, Pender C, Penaranda C, et al. Inhibition of IGF-1R and lipoxygenase by nordihydroguaiaretic acid (NDGA) analogs. Bioorg Med Chem Lett 2007; 17: 4026-9.

9 Ohshima-Hosoyama S, Hosoyama T, Nelon LD, Keller C. IGF-1 receptor inhibition by picropodophyllin in medulloblastoma. Biochem Biophys Res Commun 2010; 399: 727-32.

10 Gariboldi MB, Ravizza R, Monti E. The IGFR1 inhibitor NVP-AEW541 disrupts a pro-survival and pro-angiogenic IGF-STAT3-HIF1 pathway in human glioblastoma cells. Biochem Pharmacol 2010; 80: 455-62.

$11 \mathrm{Chu} \mathrm{CH}$, Huang CY, Lu MC, Lin JA, Tsai FJ, Tsai CH, et al. Enhancement of AG1024-induced H9c2 cardiomyoblast cell apoptosis via the interaction of IGF2R with Galpha proteins and its downstream PKA and PLC-beta modulators by IGF-II. Chin J Physiol 2009; 52: 31-7.

12 Haluska P, Carboni JM, TenEyck C, Attar RM, Hou X, Yu C, et al. HER receptor signaling confers resistance to the insulin-like growth factor-I receptor inhibitor, BMS-536924. Mol Cancer Ther 2008; 7: 2589-98.

13 Haluska P, Carboni JM, Loegering DA, Lee FY, Wittman M, Saulnier MG, et al. In vitro and in vivo antitumor effects of the dual insulin-like growth factor-I/insulin receptor inhibitor, BMS-554417. Cancer Res 2006; 66: 362-71.

14 Martins AS, Mackintosh C, Martin DH, Campos M, Hernandez T, Ordonez JL, et al. Insulin-like growth factor I receptor pathway inhibition by ADW742, alone or in combination with imatinib, doxorubicin, or vincristine, is a novel therapeutic approach in Ewing tumor. Clin
Cancer Res 2006; 12: 3532-40.

15 Liu X, Xie H, Luo C, Tong L, Wang Y, Peng T, et al. Discovery and SAR of thiazolidine-2,4-dione analogues as insulin-like growth factor-1 receptor (IGF-1R) inhibitors via hierarchical virtual screening. J Med Chem 2010; 53: 2661-5.

16 Xie H, Qin YX, Zhou YL, Tong LJ, Lin LP, Geng MY, et al. GA3, a new gambogic acid derivative, exhibits potent antitumor activities in vitro via apoptosis-involved mechanisms. Acta Pharmacol Sin 2009; 30: 346-54.

17 Guo XN, Zhong L, Zhang XH, Zhao WM, Zhang XW, Lin LP, et al. Evaluation of active recombinant catalytic domain of human ErbB2 tyrosine kinase, and suppression of activity by a naturally derived inhibitor, ZH-4B. Biochim Biophys Acta 2004; 1673: 186-93.

18 Blum G, Gazit A, Levitzki A. Development of new insulin-like growth factor-1 receptor kinase inhibitors using catechol mimics. J Biol Chem 2003; 278: 40442-54.

19 Shelton JG, Steelman LS, White ER, McCubrey JA. Synergy between $\mathrm{PI3K} /$ Akt and Raf/MEK/ERK pathways in IGF-1R mediated cell cycle progression and prevention of apoptosis in hematopoietic cells. Cell Cycle 2004; 3: 372-9.

20 Mulligan AM, O'Malley FP, Ennis M, Fantus IG, Goodwin PJ. Insulin receptor is an independent predictor of a favorable outcome in early stage breast cancer. Breast Cancer Res Treat 2007; 106: 39-47.

21 Goodwin PJ, Ennis M, Pritchard KI, Trudeau ME, Koo J, Madarnas Y, et al. Fasting insulin and outcome in early-stage breast cancer: results of a prospective cohort study. J Clin Oncol 2002; 20: 42-51.

22 Kaaks R, Lukanova A. Energy balance and cancer: the role of insulin and insulin-like growth factor-I. Proc Nutr Soc 2001; 60: 91-106.

23 Buck E, Gokhale PC, Koujak S, Brown E, Eyzaguirre A, Tao N, et al. Compensatory insulin receptor (IR) activation on inhibition of insulinlike growth factor-1 receptor (IGF-1R): rationale for cotargeting IGF-1R and IR in cancer. Mol Cancer Ther 2010; 9: 2652-64.

$24 \mathrm{Kim}$ KS. Multifunctional role of Fas-associated death domain protein in apoptosis. J Biochem Mol Biol 2002; 35: 1-6.

25 Green DR, Reed JC. Mitochondria and apoptosis. Science 1998; 281: 1309-12.

26 Gossage L, Eisen T. Targeting multiple kinase pathways: a change in paradigm. Clin Cancer Res 2010; 16: 1973-8.

27 Iyer R, Fetterly G, Lugade A, Thanavala Y. Sorafenib: a clinical and pharmacologic review. Expert Opin Pharmacother 2010; 11: 194355.

28 Goodman VL, Rock EP, Dagher R, Ramchandani RP, Abraham S, Gobburu JV, et al. Approval summary: sunitinib for the treatment of imatinib refractory or intolerant gastrointestinal stromal tumors and advanced renal cell carcinoma. Clin Cancer Res 2007; 13: 1367-73.

29 Lin LG, Xie H, Li HL, Tong $\sqcup$, Tang CP, Ke CQ, et al. Naturally occurring homoisoflavonoids function as potent protein tyrosine kinase inhibitors by c-Src-based high-throughput screening. J Med Chem 2008; 51: 4419-29. 